\title{
ASSM: Agent Based Source Specific Multicast Routing Protocol for Wireless Mesh Networks
}

\author{
Mojtaba Khayat
}

\begin{abstract}
Wireless mesh networks (WMNs) are new emerging networks which are anticipated to resolve many limitation of ad-hoc networks, sensor networks and wireless local area networks and improve their performance. But still there are many unresolved research challenge in this area. In this paper we have proposed source-specific multicast protocol for wireless mesh network, which has many application in, multimedia, radio and TV multicasting and distance learning. We have used core-based approach to construct minimum cost tree (MCT) among member nodes and optimized this tree for multiple metrics by applying ant colony optimization metaphor.
\end{abstract}

Index Terms-Wireless mesh networks (WMNs);multicast routing; ant colony optimization

\section{INTRODUCTION}

Wireless mesh network (WMNs) consist of mesh routers and mesh clients, mesh client can be stationary or mobile while mesh router have minimum mobility. WMNs have the capability of self-forming; self healing, and selforganization, moreover, the gateway functionality in mesh routers enable the integration of WMNs with various existing wireless networks, such as Internet, IEEE 802.11, etc. [1]

Any-source multicast (ASM) is IP multicast service model which the IP datagram is transmitted to a set of zero ore more end-host Identified by single IP address G, Endhosts may join and leave the group any time and there is no restriction on their numbers. Also any end-host may transmit to a host group, even if it's not a member of that group [2]. In the other hand, Specific-source multicast (SSM) is IP multicast service model which the IP datagram is transmitted by a source S to an SSM destination address G, and receivers can receive this datagram by subscribing to channel (S,G) [2]. SSM support many important applications such as distribution of software, IP Radio and TV multicasting and distance learning. In SSM scenario end-hosts express interest in receiving data from specified source $\mathrm{S}$ of multicast group $\mathrm{G}$, therefore the source address is known priori.

There are two main approaches to multicast routing; Treebased and Mesh-based approaches. In tree-based multicasting shortest path tree(SPT) or minimum cost tree(MCT) minimize the distance or cost from sender to receivers, while in mesh-based multicasting multiple path between any source and receiver pair may exist to increase robustness [3]. Tree-based approaches are a well established

Manuscript received February 29, 2012; revised April 27, 2012. This work was supported by computer department of Islamic Azad University, Iran.

M. khayat works in the Azad University. Wireless Mesh Networks and Ad hoc are his research fields. concept in wired networks, SPT tree try to minimize the distance between the source and each receiver therefore it will consequently minimize the end to end delay. On the other hand MCT tree is based on minimum Steiner tree (MST) which is NP-Hard problem [3]. For wireless networks, the MCT cost is redefined in [4]. Because in wireless network the transmission of multicast packet to any number of neighbor can be done by single transmission, minimum cost tree is one which connect the source and receivers with minimum number of transmission. Later in this work we will define multicast efficiency of routing protocol by using this concept. In contrast to tree-based approaches mesh-based approaches may have more than one path between any source and receiver pair. This approach has benefit in MANET where network topology change frequently [5], therefore having multiple path increase the chance of datagrams to be delivered to the receiver. There is also a hybrid approach to multicasting in wireless networks. While tree-based approaches consider mainly performance and mesh-based approaches focus on robustness, hybrid approaches try to use advantage of both tree and mesh to achieve both performance and robustness simultaneously.

Swarm intelligence is a relatively new approach to problem solving that takes inspiration from the social behaviors of insects and of other animals. Ant colony optimization is one of the successful swarm intelligence methods in general purpose optimization. Real ants deposit pheromone on the ground in order to mark some favorable path that should be followed by other members of the colony. Ant colony optimization exploits a similar mechanism for solving optimization problems. One of the first applications of ACO in routing and load balancing in packet switched network is ANTNET [6], which inspired this work as well.

We will present a novel agent based multicast protocol in this paper. We have used core-based approach to construct minimum cost tree (MCT) among member nodes and optimized this tree for multiple metrics by applying ant colony optimization metaphor. In section II we will present an overview of ASSM multicast protocol, in section III we will present some simulation scenario and results and in section IV we will conclude this paper.

\section{OVERVIEW OF ASSM PROTOCOL}

We will present Source-Specific Multicast (SSM) protocol for wireless mesh network in this section. Each mobile node in the network will communicate with on of the mesh routers directly, and all mobile nodes previously knows multicast source address. We will neglect mobile nodes in our simulation and assume traffic is generated from the mesh routers which cover the sources and ends in mesh 
routers which cover the destination nodes. Therefore throughout this paper we use the word node and mesh router interchangeably.

We will use tree topology with core node which is the multicast source of the group. For each multicast group, each forwarder of the group knows its head node, which is the node it receives packets coming from the group source. Also each forwarder have a list of its down stream nodes which are neighbor nodes registered to receive multicast packets of this group. A forwarder must have at least one neighbor node which is registered to receive multicast packets or it should withdraw from being a forwarder. For each multicast group pair $(\mathrm{S}, \mathrm{G})$ all node of the network store

- A probabilistic vector $\mathrm{V}$ for routing forward agent of this group

- Local routing information; which is mean and variance of each of routing metrics over a moving observation window $\mathrm{W}$.

- Head node address $\mathrm{H}$; which is a neighbor node from which this node receives multicast traffic coming from the source of the group.

- List of neighbor nodes registered to receive multicast traffic transmitted by this node.

As discussed earlier in SSM each multicast group is represented by source and group id pair $(\mathrm{S}, \mathrm{G})$. When a node decides to join a multicast group it will launch a forward agent toward a core node (Source of multicast group). The forward agent at each node will choose the next hop randomly with the aid of probabilistic routing table (which is a table of probabilistic column vectors for each destination) and save the visited node id in its memory. When finally a forward agent reaches the core of the group it will launch a backward agent, transfer its memory to this new agent and die. The backward agent will travel the reverse path of the forward agent and will update routing table probabilities for reaching the core at each node. When backward agent reaches the original sender of the forward agent, the node learns a path toward the group source. If the new path is the first path which the node has discovered so far or if it outperform current path by specified margin, the node will use this new path. When a node decides to use a new path it launches a reserve agent toward the core and transfer backward agent memory to this agent. Reserve agent will inform nodes along the path that they should become a forwarder of this group. If the node which launches the reserve agent currently uses another path it will launch a release agent to its head node to inform that this node is not required to be a forwarder anymore. When a member of multicast group wants to withdraw from that group it launches a release agent to its head node and it will not launch any other forward agent toward the core of the group.

\section{A. Hello Messages}

Each node at constant period of time will broadcast a hello message to inform its neighbors about its presence. Each node will have a list of all its neighbors and after a specified period of time if it does not receives a hello message from one of its neighbors, it will consider that the neighbor node has moved away or no longer operational and therefore it will remove this neighbor from the list.

\section{B. Local Routing Information}

At each node of the network for each multicast group pair (S, G) we will store routing statistic information for reaching this group core. At each node, this routing information are provided by backward agents and includes delay from core to this node and number of nodes which are not a forwarder of this group on the path traveled by the backward agent so far. Delay and number of non-forwarding nodes are metrics we are using in this routing mechanism and the goal is to choose a path from the set of all available paths which minimize both of these metrics. For each metric mean and variance of samples (i.e. provided by backward ants) are stored in network nodes for each multicast group and is called local routing information. When a backward agent enters a node the value of these metrics provided by agent is compared by local routing information and the reinforcement value of agent path is computed. Reinforcement value $r$ which is dimensionless value $r \in[0$, 1] represents goodness of backward agent trip path estimated by use of local routing information.

$$
\begin{gathered}
r_{d}=r_{d}(d, \mu, \sigma) . \\
r_{n}=r_{n}(n, \mu, \sigma) . \\
r=r\left(r_{d}, r_{n}\right) .
\end{gathered}
$$

This value is used as reinforcement for choosing previous hop which backward agent comes from as a next hop toward group core. After computing reinforcement value local routing information is updated by these new values. Such that

$$
\begin{gathered}
\mu=\mu+\eta(o-\mu) . \\
\sigma^{2}=\sigma^{2}+\eta\left((o-\mu)^{2}-\sigma^{2}\right) .
\end{gathered}
$$

where $\eta$ is $5 / \mathrm{W}$, and $\mathrm{W}$ is the size of observation window.

\section{Forward Agents}

When a node decides to join a multicast group it start to launch forward agents toward multicast group core in specified time intervals. A forward agent will use a probabilistic vector of its multicast group at each node to choose the next hop and randomly travel through the network nodes until it reaches the group core. When a forward agent passes a node stores a node id in its memory and after reaching the core will pass its memory to backward agent.

Time to live field in forward agent packets can be set according to network dimension so that forward agents that can not manage to reach the destination in appropriate time and are wondering around the network are discarded automatically.

\section{Backward Agents}

When forward agent reaches the core of the multicast group it will launch a backward agent toward a node how wants to join to the group and transfer its memory to this 
agent and die. Backward agent travel the reverse path traveled by forward agent and at each intermediate node and at the destination update the local routing information and probabilistic vector of this multicast group.

\section{E. Probabilistic Routing Vector}

For each multicast group pair $(\mathrm{S}, \mathrm{G})$ all node of the network store a vector $\mathrm{V}$ whose dimension is $\mathrm{N}$ (where $\mathrm{N}$ is number of neighbor of the node). Each element of $\mathrm{V}$ represents goodness of choosing respected neighbor as the next hop in probabilistic fashion, when destination is multicast group source $\mathrm{S}$. when a forward agent enter a node it will choose the next hop toward group core randomly from set of all current neighbors of the node except its previous hop. Forward agent uses probabilistic vector in such a way that the neighbor with higher value will have a higher chance of being selected as a next hop. When backward agent enter a node, reinforcement value of choosing the neighbor node which backward agent come from will be calculated by using agent data and local routing information. Then this reinforcement value will be used to update probabilistic vector. The probability of choosing node $\mathrm{n}$ as next hop will increase as follow

$$
P_{n} \leftarrow P_{n}+r\left(1-P_{n}\right) \text {. }
$$

And probabilities of choosing all other neighbors such as $f$ will be decreased so that the sum of all probabilities remain one

$$
P_{f} \leftarrow P_{f}-r P_{f}
$$

\section{F. Reserve Agents}

After receiving the backward agent the member node will switch to new discovered path if probability value of using the neighbor node which backward agent comes from outperforms the current head probability value by specified margin. When a node considers joining a group using a path provided by backward agent, it will launch a reserve agent and the backward agent memory will be transferred to this agent. Reserve agent will travel a reverse path traveled by backward agent until it reaches a group forwarder or the group core. When a node receives a reserve agent of a multicast group, it will set the flag to forward the data packet for this group. Also it will register the previous hop of the reserve agent in the list of receiving nodes of this group. The next hop of the reserve agent will become the head node of this multicast group at current node and then the reserve agent will be forwarded to next hop on its memory. If the node which receives a reserve agent was already a forwarder of this group or it was a group core, the previous hop of the reserve agent will be registered on receiving node list, but the head address of this group will not changed and the reserve agent will die.

\section{G. Release Agent}

When a multicast group member considers using another path or wants to withdraw from receiving multicast packets. It launches a release agent to its head node. Upon receiving a release agent forwarder node removes the sender from the list of receiving neighbors of this multicast group and the release agent dies. If a list of receiving neighbors of multicast group forwarder becomes empty, it will not forward this group data packets anymore. In addition if the forwarder node itself dose not be a member of this group it will launch a release agent to its head node. The group core itself will not transmit any multicast data if the list of its receiving neighbor becomes empty.

\section{RESUlts AND Discussion}

This protocol is implemented in a discrete event simulator written in $\mathrm{C}++$. In order to evaluate the performance of protocol we have used following metrics

- Packet delivery ratio. The number of data packet delivered to receivers versus the number of data packets supposed to be received.

- Multicast efficiency. Total data bytes delivered to member nodes versus total data bytes transmitted by all forward nodes.

- Routing overhead. Total routing agent bytes transmitted by network nodes versus total data bytes delivered to member nodes.

- Average end to end delay. The average of end to end delay of every data packet delivered for all data packets.

- Number of forwarder nodes. The number of forwarding nodes in multicast tree.

We have simulated a simple network of 16 nodes shown in fig. 1 consisting of one multicast group with source located at node 0 and three receiver nodes (nodes 7, 13 and 15) .

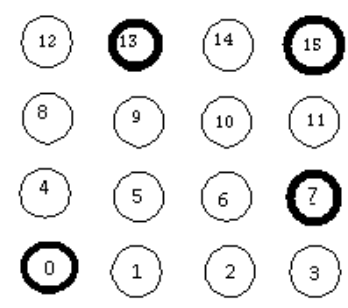

Fig. 1. Simple network topology

TABLE I: Simple NETWORK SIMULATION PARAMETERS

\begin{tabular}{ll}
\hline \multicolumn{1}{c}{ Parameter } & \multicolumn{1}{c}{ Value } \\
\hline Network size & 16 nodes over $600 \mathrm{~m} * 600 \mathrm{~m}$ area \\
Network topology & Orthogonal grid \\
Multicast group size & 3 receivers \\
Wireless antenna range & $250 \mathrm{~m}$ \\
Transmission rate & $11 \mathrm{Mbit} / \mathrm{s}$ \\
Packet size & 512 bytes \\
Source traffic model & Constant bit rate $(\mathrm{CBR})$ \\
Multicast sender rate & 10 packets $/ \mathrm{s}$ \\
Simulation time & $100 \mathrm{~s}$ \\
Hello interval & $10 \mathrm{~s}$ \\
Agent launching rate & 2 agent/s \\
\hline
\end{tabular}

Table I shows simulation parameters for this network and fig 1 shows a schematic of network topology. The path with minimum forwarder nodes for current situation contain 6 nodes and the simulation results show that if we only optimize the number of forwarder nodes this protocol will use such path after about 40 second from start of the simulation

Fig. 2 shows multicast efficiency for performance (i.e. we have considered only number of forwarders metric) and performance and delay (i.e. we have considered both routing 
metric). As can be seen in this figure for performance case multicast efficiency is about 0.5 after 40 second from the start of simulation. If we use both routing metrics delay and number of forwarders, multicast efficiency will decrease but in fig. 3 we can observe that average delay will decreased too. It's obvious that delay and multicast efficiency cannot be minimized in the same time therefore protocol will find the best path according to weights we have assigned to each of these metric in calculation of reinforcement value.

For better evaluation of proposed protocol we have simulated medium size network adopted one of scenarios from [3] and compared the result with the result presented in this reference. Table 2 shows the simulation parameter for this simulation. Fig. 4 show the number of forwarder for different number of receivers and fig. 5 shows average path length for different number of receivers for this network.

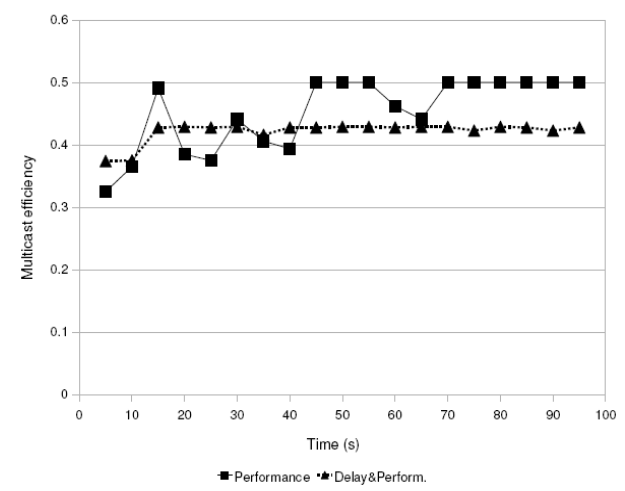

Fig. 2. Multicast efficiency for simple network

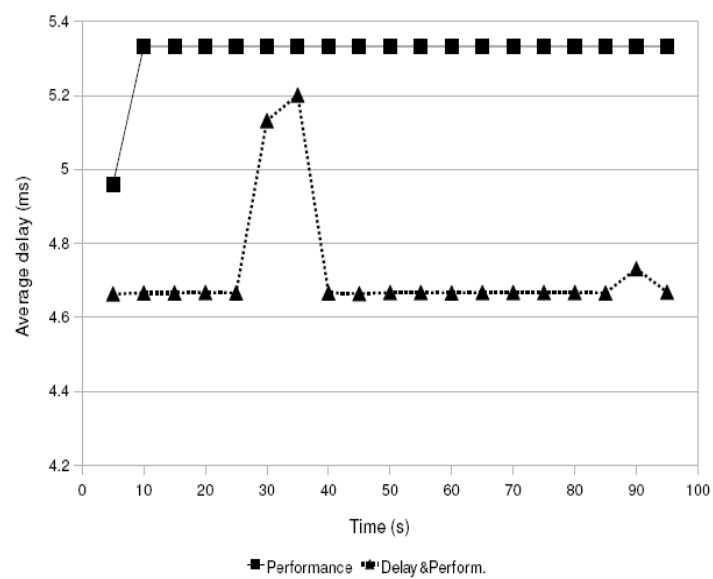

Fig. 3. Average delay for simple network

From fig 4 and fig 5 we can conclude that the performance of this protocol is much like MNT and MST but the point is that in all these simulation we have used 0.7 for number of forwarders weight and 0.3 for delay weight in calculation of reinforcement value. Therefore if we adjust the weights toward the delay we will get closer to the SPT, results. But the important point here is that we have a protocol that we can tune it according to our needs.

\section{CONCLUSION AND FUTURE WORK}

We have presented a novel agent based source specific multicast protocol in this paper. We have used core-based approach to construct minimum cost tree (MCT) among member nodes and optimized this tree for multiple metrics by applying ant colony optimization metaphor. Using swarm intelligence for path optimization has paved the way for optimization any number of metrics simultaneously while discovering new paths. In this work we have used weighted sum of two network metric; delay and number of nonforwarding node on new paths to calculate reinforcement value, but we can extend these metric to include bandwidth, jitter or even security metrics.

TABLE II: MEDIUM SIZE NETWORK SIMULATION PARAMETERS

\begin{tabular}{ll}
\hline \multicolumn{1}{c}{ Parameter } & \multicolumn{1}{c}{ Value } \\
\hline Network size & 100 nodes over $2000 \mathrm{~m} * 2000 \mathrm{~m}$ area \\
Network topology & Uniform random distribution \\
Multicast group size & $\{20,40,60\}$ receivers \\
Wireless antenna range & $250 \mathrm{~m}$ \\
Transmission rate & $11 \mathrm{Mbit} / \mathrm{s}$ \\
Packet size & 512 bytes \\
Source traffic model & Constant bit rate $(\mathrm{CBR})$ \\
Multicast sender rate & 10 packets $/ \mathrm{s}$ \\
Simulation time & $300 \mathrm{~s}$ \\
Hello interval & $10 \mathrm{~s}$ \\
Agent launching rate & $5 \mathrm{agent} / \mathrm{s}$ \\
\hline
\end{tabular}

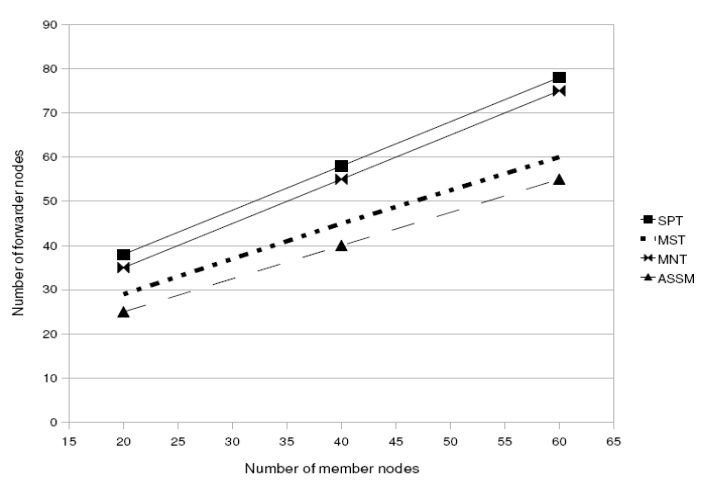

Fig. 4. Number of forwarder nodes for different number of receivers

This opportunity in choosing any number of desired routing metric is one of the main differences of this work and other wireless multicast protocols which are presented in literatures. One of the main features of present work is its independence from underlying unicast protocol therefore any unicast protocol can be used for that purpose. This work is in its beginning stage and we should consider many other factor to present a complete and mature multicast routing protocol in WMNs, in the other hand we have not optimized any of the routing parameter in the presented simulation and still results show presented method could be a promising approach to multicasting in WMNs.

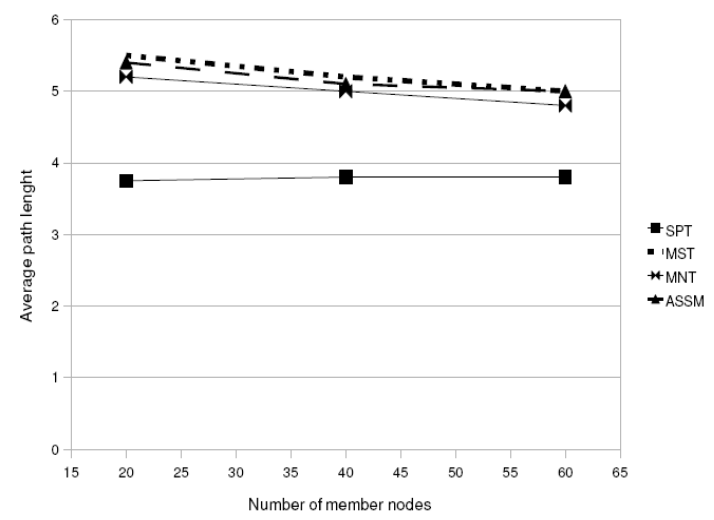

Fig. 5. Average path length for different number of receivers 


\section{REFERENCES}

[1] I. F. Akyildiz, X. Wang, and W. Wang, "Wireless mesh network: a survey," Computer Networks, vol. 47, pp. 445-487, 2005.

[2] S. Bhattacharyya and E. d. Sprint, "An Overview of Source-Specific Multicast (SSM)," 2003.

[3] U. T. Nguyenl, "On multicast routing in wireless mesh networks," Computer Communications, vol. 31, pp. 1385-1399, 2008.
[4] P. M. Ruiz and A. F. Gomez-Skarmeta,"Approximating optimal multicast trees in wireless multihop networks," in IEEE Symposium on Computers and Communications, pp. 686-691, 2005.

[5] C. d. M. Cordeiro, H. Gossain, and D. P. Agrawal, "Multicast over wireless mobile ad hoc networks: present and future directions," IEEE Network, vol. 17, pp. 52-59, 2003.

[6] G. D. Caro and M. Dorigo, "AntNet: Distributed Stimergic Control for Communications Networks," Journal of Artificial Intelligence Research, vol. 9, pp. 317-365, 2003. 